\title{
Ascaris lumbricoides infection: an unexpected cause of pancreatitis in a western Mediterranean country
}

\author{
A. Galzerano, ${ }^{1}$ E. Sabatini ${ }^{7}$ and D. Duri ${ }^{2}$
}

\section{Introduction}

Ascaris lumbricoides is a nematode parasite, endemic in the Middle East and South America, especially in rural countries. Ascariasis infection causes about 20000 deaths every year [1], usually as a result of intestinal occlusion, and it contributes to infant malnutrition [2]. Poor sanitation is usually the most important risk factor for infection, and women are more affected because progesterone plays a role in inducing Oddi's sphincter relaxation, allowing the nematode to access the biliary duct [3]. Although not common in developed countries, ascariasis infection is increasingly likely to be encountered by clinicians because of the growing rates of travel to developing countries and increased migration.

\section{Case report}

We describe the case of a 78-year-old Italian woman who had never travelled abroad, who was admitted to the surgical ward of A. Murri Hospital, Fermo, Italy, with fever (temperature $38^{\circ} \mathrm{C}$ ), leukocytosis (white blood cell count $\left.15.4 \times 10^{3} / \mu \mathrm{L}\right)$, hyperamylasaemia (serum amylase level $260 \mathrm{U} / \mathrm{L}$ ) and abdominal pain.

The patient underwent abdominal ultrasonography and a computerized tomography (CT) scan of the abdomen and thorax, which revealed peritoneal effusion, pancreatic oedema, dilated gallbladder with a bile duct measuring $1.1 \mathrm{~cm}$ with no lithiasis, left pleural effusion and basal atelectasis. Endoscopic retrograde cholangiopancreatography showed a dilated bile duct with a patent ampulla with no lithiasis.

The day after admission the patient underwent cholecystectomy, cholangiogram, positioning of Kher drainage and pancreatic necrosectomy. Due to haemodynamic instability and respiratoryfailurethe patient wasthenadmitted to the intensive care unit. At admission she was apyretic and microbiological cultures from abdominal drainage specimens were negative. After weaning and extubation the patient was transferred to the surgical ward where she underwent an unremarkable recovery.

About 20 days after admission she developed fever, nausea, vomiting, marked eosinophilia that had not been noticed before (total leukocyte count $11.8 \times 10^{3} / \mu \mathrm{L}$, eosinophils $10 \%$ ) and a maculopapular rash. On the hypothesis of iatrogenic allergic dermatitis, steroid and antihistamine treatments were started, with no benefit.

One week later the patient vomited a $5 \mathrm{~cm}$ male ascarid nematode. Therapy with mebendazole $100 \mathrm{mg}$ twice daily was started with prompt resolution of the pancreatic oedema, as documented by CT scans. The patients' subsequent recovery was uneventful and she was discharged 48 days after initial admission.

\section{Discussion}

Ascaris lumbricoides infestation is acquired through ingestion of eggs in raw vegetables. The human is the definitive host. Ingested larvae penetrate the intestinal lymphatic and venous vessels and through the portal vein reach the right heart, pulmonary circulation and the alveoli. After alveolar rupture they pass into the trachea and the pharynx, are then swallowed; after about 2 months they reach maturity. In the bowel nematodes can perforate the intestinal wall, be ejected from the mouth or anus and penetrate the biliary ducts or the airways. The infestation can present as a wide range of symptoms: intestinal perforation or occlusion, cholangitis, obstructive jaundice, acute pancreatitis or appendicitis, pneumonia and respiratory failure and allergic reactions to the ascaris antigen. In most cases, however, patients present with unspecific symptoms and sometimes the diagnosis is incidental [3].

The diagnosis is usually made by abdominal ultrasonography, revealing biliary duct dilation and the presence of the parasite, a hyperechoic linear structure with a hypoechogenic line inside, which is sometimes motile [3-5]. Ultrasonography is also the gold standard technique for follow-up. CT scan and nuclear magnetic resonance imaging can also be helpful. Endoscopic retrograde cholangiopancreatography is the gold standard method for

${ }^{1}$ Department of Anaesthesia and Intensive Care, Santa Maria della Misericordia Hospital, Perugia, Italy.

${ }^{2}$ Department of Anaesthesia and Intensive Care, Sant Antonio Hospital, San Daniele del Friuli, Italy (Correspondence to D. Durì: dav.anestesia@ gmail.com).

Received: 13/01/08; accepted: 09/03/08 
identifying and removing the nematode from the duodenal, biliary or pancreatic tract [3].

In this case neither CT scans nor endoscopic retrograde cholangiopancreatography was able to reveal the presence of the parasite, which probably had already migrated to the left lung, causing basal atelectasis and pleural effusion at the time the examinations were made.

Although ascariasis is the most common human worm infection in the Mediterranean area, the development of a severe illness such as a pancreatitis due to this infestation is unusual [6-9]. The origin of the infestation was not established. As the patient had not travelled to any endemic areas, our hypothesis is a contact with eggs through consumption of raw vegetables or contaminated soil. The presence of eosinophilia should have raised suspicion of the possibility of a parasitic infection, even in a patient not travellingormigratingfromendemic areas, but the rarity of this cause of acute abdomen was certainly misleading.

\section{References}

1. Khuroo MS. Ascariasis. Gastroenterology clinics of North America, 1996, 25:553-77.

2. Villamizar E et al. Ascaris lumbricoides infestation as a cause of intestinal obstruction in children: experience with 87 cases. Journal of pediatric surgery, 1996, 31:201-4.

3. Misra SP, Dwivedi M. Clinical features and management of biliary ascariasis in a non-endemic area. Postgraduate medical journal, 2000, 76:29-32.

4. Hoffmann $\mathrm{H}$ et al. In vivo and in vitro studies on the sonographical detection of Ascaris lumbricoides. Pediatric radiology, 1997, 27:226-9.

5. Ferreyra NP, Cerri GG. Ascariasis of the alimentary tract, liver, pancreas and biliary system: its diagnosis by ultrasonography. Hepatogastroenterology, 1998, 45:932-7.

6. Petit A et al. L'ascaridiose: une cause d'angiocholite peu banale sous nos climats [Ascariasis: an unusual cause of cho- langitis in our climate]. Gastroentérologie clinique et biologique, 1991, 15:660-1.

7. Moulinier C, Battin J, Giap G. Evolution du taux de prevalence de quatre parasites intestinaux chez l'enfant [Development of the prevalence rate of four intestinal parasites in children]. Pediatrie, 1990, 45:129-32.

8. Mosiello G et al. Ascaridiasi come possibile causa di addome acuto anche in Italia: presentazione di un caso clinico [Ascariasis as a cause of acute abdomen: a case report]. $L a$ pediatria medica e chirurgica, 2003, 25:452-4.

9. De la Cruz Alvarez J et al. Ascariasis biliopancreática: una entida infrecuente en nuestro medio [Biliopancreatic ascariasis: an infrequent disease in our environment]. Gastroenterología y hepatología, 1996, 19:210-2. 